\title{
Cloud Operations
}

\author{
Hans-Peter Fröschle • Nikolaus Schmidt
}

(C) Springer Fachmedien Wiesbaden GmbH, ein Teil von Springer Nature 2020

Die Nutzung von IT-Leistungen aus der Cloud ist in den letzten Jahren kontinuierlich gestiegen. Kennzeichnend dabei ist, dass neben den klassischen Entscheidungsgründen wie Kosteneffizienz, flexible und schnelle Skalierbarkeit und ortsunabhängiger Zugriff aktuelle Cloud-Lösungen mehr und mehr als Basis für die digitale Transformation von Geschäftsmodellen und Unternehmen in den Fokus rücken. CloudLösungen unterliegen damit einem Paradigmenwechsel von eher outsourcing-orientierten Entscheidungen hin zu strategischen Entscheidungen bei der Cloud-Nutzung. Neben der Digitalisierung bestehender Geschäftsprozesse ist hier vor allem auch die Neuentwicklung innovativer Geschäftsmodelle zu erwähnen. Bereits in der Vergangenheit wurde diese Entwicklung als absehbarer Paradigmenwechsel vom reinen Outsourcing mittels Cloud-Lösungen hin zu Cloud-Sourcing mit neuen Potenzialen prognostiziert. Schnelles Prototyping von cloud-basierten Anwendungen, der Aufbau der organisationsweiten Cloud-Plattformen sowie die Migration von Applikationen auf diese Plattformen standen dabei bisher im Fokus.

Cloud-Lösungen haben inzwischen in vielen Organisationen einen ersten Reifeund Nutzungsgrad erreicht. Damit rücken nun verstärkt Themen um den Betrieb und die kontinuierliche Weiterentwicklung von Cloud-Plattformen in den Vordergrund. Damit verändern Cloud-Lösungen nicht nur die Art und Weise, wie die Unternehmen IT-Services beziehen, sondern auch wie diese Cloud Services genutzt werden. Cloud-Lösungen kommen in diesem Stadium vor allem zum Einsatz, um Kosten für die eigene IT-Infrastruktur einzusparen bzw. um benötigte IT-Infrastruktur-Kapazitäten bedarfsorientiert flexibel nutzen zu können. Individuelle Lösungen widersprechen dabei allerdings dem Geschäftsmodell der Cloud Provider nach einer kundenübergreifenden, hohen Skalierbarkeit der bereitgestellten Services. Die

H.-P. Fröschle ( $\square)$

i.t-consult GmbH, Pischekstraße 68, 70184 Stuttgart, Deutschland

E-Mail: hpf@i-t-consult.de 
Nutzung von Cloud-Lösungen setzt somit einen hohen Standardisierungsgrad der Leistungserbringung im IT-Betrieb voraus. Die Unternehmens-IT muss von einer bislang individualisierten, handwerklich geprägten Leistungserbringung auf eine industrielle, fabrikorientierte Leistungserstellung umgestellt werden. Gewachsene, OnPremise-Architekturen müssen an gängige Architekturen der Cloud Provider angepasst werden. Dies ist vor allem für Unternehmen eine zentrale Herausforderung, die in der Vergangenheit eine Vielzahl, teilweise komplexer Eigenentwicklungen betrieben haben. Abgesehen von isolierten IT-Services, die über eine Cloud-Lösung betrieben werden, erfordern IT-Services, die mit anderen betrieblichen IT-Services interagieren beispielsweise die Anpassung von Monitoring-, Authentifizierungs- und Berechtigungskonzepten.

Neben der Standardisierung und Optimierung der technischen Prozesse im ITBetrieb ist die Standardisierung des IT-Servicemanagements eine weitere Voraussetzung zur Nutzung von Cloud-Lösungen. Vor einem Outsourcing von IT-Services in die Cloud muss sichergestellt sein, dass der Betrieb der Cloud-Plattformen und -Services in der betrieblichen Praxis reibungslos funktioniert und organisatorische Standards in Bezug auf Security, Datenschutz und Servicemanagement erfüllt sind.

So ist beispielsweise die Standardisierung von Schnittstellen zwischen den im Unternehmen und beim Cloud Provider eingesetzten Servicemanagement-Tools eine wesentliche Anforderung, um eine strukturierte und nachvollziehbare Bearbeitung von Incidents, Changes und Problems über alle Eskalationsstufen hinweg gewährleisten zu können. Ebenso ist zu überlegen, wie die flexible Bereitstellung und nutzungsbasierte Abrechnung von Cloud Services über ein entsprechend angepasstes Service Request Management bzw. weitgehend automatisierte Bestellprozesse optimiert werden können.

Einen qualitativen Sprung bei der Nutzung von Cloud-Lösungen können Unternehmen dann realisieren, wenn sie mit Cloud Services ihre technologischen Prozesse im Hinblick auf Flexibilität und Agilität des gesamten Unternehmens optimieren und die Basis für neue Geschäftsmodelle legen. Der Begriff Cloud Operations beschreibt hierbei alle organisatorischen, prozessualen sowie technischen Lösungen, welche für den Betrieb von cloud-basierten Applikationen sowie Plattformen in einer Organisation erforderlich sind.

Der einführende Beitrag zeigt den aktuellen Stand von Forschung und Entwicklung zum Thema Cloud Operations auf und gibt erste Umsetzungshinweise aus der betrieblichen Praxis. Die folgenden Beiträge verdeutlichen, dass ein agiler, cloudbasierter IT-Betrieb die Voraussetzung für weitergehende IT-Themen wie agile Software-Entwicklung ist. Anforderungen und Lösungen für das Provider Management, Vorgehensmodelle zur Einführung und Nutzung von Cloud Services, Rahmenbedingungen der Cloud-Nutzung für kleine und Kleinstunternehmen werden ebenso diskutiert, wie Beispiele cloud-basierter Services zur Bewältigung der digitalen Transformation unter den Bedingungen der Corona-Pandemie.

Drei Rezensionen zu Überblicks- und vertiefenden Themen ergänzen die Beiträge des Schwerpunktheftes. 
Unseren Autoren möchten wir an dieser Stelle einen herzlichen Dank aussprechen, dass das Heft mit den vorliegenden Inhalten trotz erschwerter Bedingungen in einer Pandemie-Situation entstehen konnte.

Wir wünschen Ihnen eine spannende Lektüre und freuen uns über Ihr Lob, aber auch über Anregungen und Kritik.

Hans-Peter Fröschle und Dr. Nikolaus Schmidt 